Archive for

Organic Chemistry

Arkivoc 2020, part vii, 353-364

\title{
A-ring functionalization of cholestane with highly substituted pyrans and 2- aminoisophthalonitriles
}

Hélio M. T. Albuquerque, Telmo Francisco, Daniela Malafaia, José A. S. Cavaleiro, and Artur M. S. Silva* LAQV-REQUIMTE, Department of Chemistry, University of Aveiro, Campus de Santiago, 3810-193 Aveiro, Portugal

Email: artur.silva@ua.pt

Dedicated to Prof. Jan Bergman on the occasion of his $80^{\text {th }}$ anniversary

Received 11-01-2020

Accepted 02-02-2021

Published on line $02-08-2021$

\section{Abstract}

Following our ongoing interest on modified steroidal compounds for biological purposes, herein we endeavor the two-step regioselective synthesis of three-substituted pyran-cholestane fused compounds (6 examples, yields up to $83 \%)$ at C-2:C-3 A-ring side of the steroid scaffold. In the presence of higher amounts of malononitrile, the intermediate $\alpha, \beta$-unsaturated carbonyl substrates give rise to competing reactions leading to the formation of the corresponding 2-aminoisophthalonitrile fused compounds ( 2 examples, up to $37 \%$ yield).

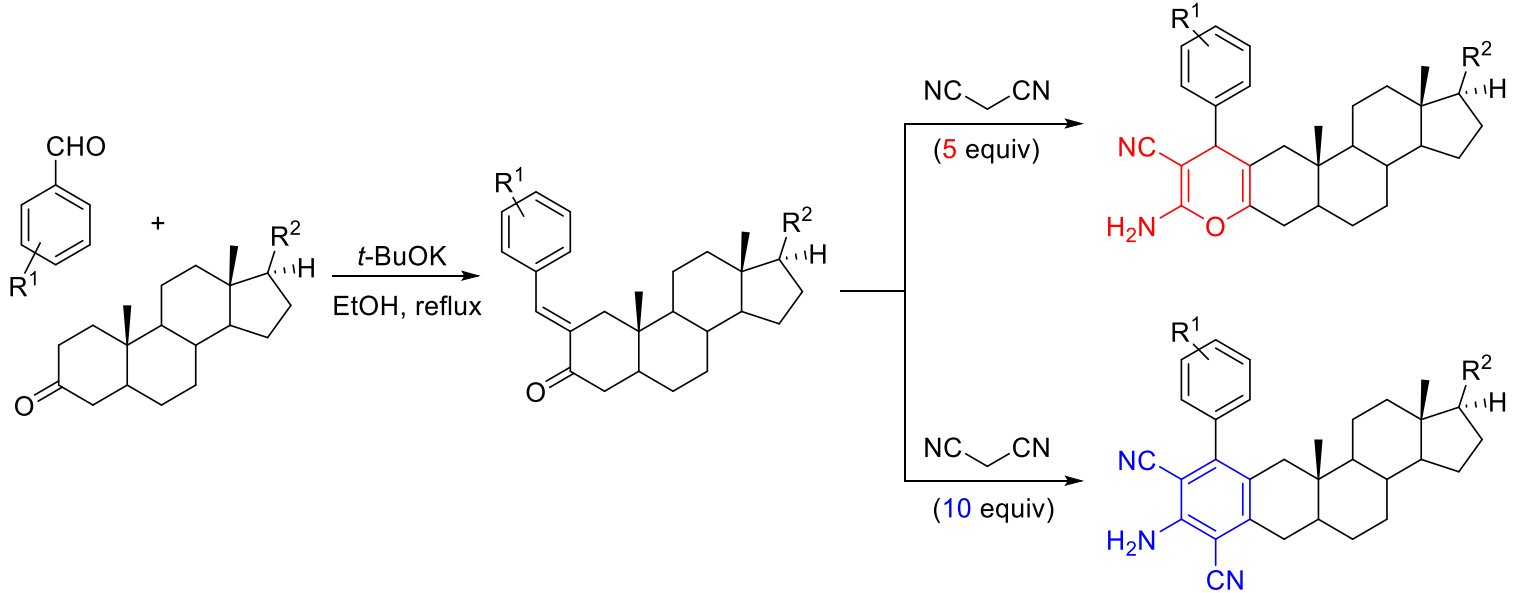

Keywords: Steroids, cholestane, substituted pyrans, 2-aminoisophthalonitriles, hybridization 


\section{Introduction}

Steroid compounds are widely present in living organisms playing important roles in their vital activities. The most recognizable steroid is cholesterol (cholest-5-en-3ß-ol), which is considered a lipid-type molecule, being one of the most important structural components of cell membranes. Cholesterol is a rigid and almost planar molecule with a steroidal skeleton of four fused rings, three six-membered and one five-membered, conventionally lettered from $A$ to $D$ (1,2-cyclopentanoperhydrophenanthrene ring system) (Figure 1).
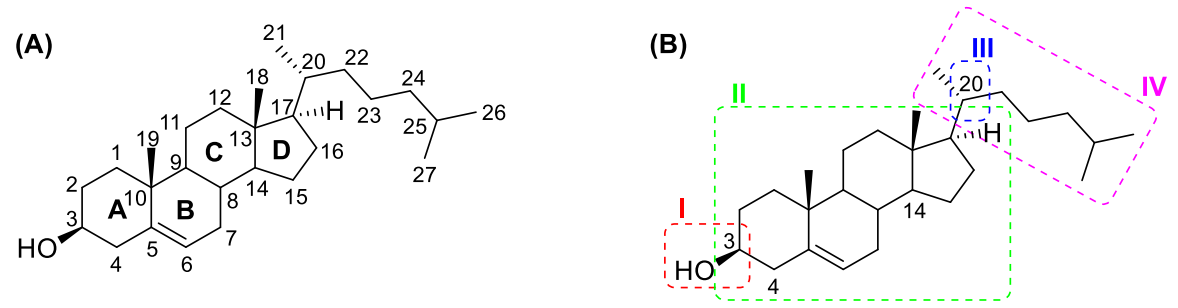

Figure 1. (A) Numbering and ring-labeling convention in cholesterol; (B) four domains of cholesterol.

The cholesterol molecule contains four essential domains (Figure 1B). In domain I, the polarity of the 3hydroxy group constitutes an active site for hydrogen bond interactions with a variety of biological molecules. ${ }^{1}$ In domain II, the absence of methyl groups at C-4 and C-14 influences directly the planarity of the molecule, while in domain III, the natural $(R)$-configuration at C-20 determines the "right-handed" conformation of the side chain. Finally, in domain IV, the conformation and length of the side chain is of prime relevance to intermolecular contacts. ${ }^{2}$ The unique properties of cholesterol make it a very attractive molecule for synthetic organic studies, as well as to other interesting applications such as drug delivery and bioimaging applications, and even liquid crystals and gelators. ${ }^{3}$ A key structural feature is the presence of the hydrophilic 3-hydroxy headgroup on the A-ring, together with a hydrophobic hydrocarbon body, which offer the molecule an amphiphilic nature, and revealed itself as being an important property in the interaction with protein aggregates. In fact, lanosterol and 25-hydroxycholesterol (Figure 2) were reported to reverse crystallininduced protein aggregation, by dissolving aggregated proteins from amyloid-like fibrils. Due to their amphiphilic nature, they were able to intercalate and coat hydrophobic core areas of large protein aggregates, allowing these aggregates to become water-soluble again. ${ }^{4,5}$ The efficacy of lanosterol to reverse multiple types of mutant-crystallin aggregates was further improved trough 2-fluorination and 25-hydroxylation, enhancing the anti-aggregating activity (Figure 2). ${ }^{6}$ Remarkably, lanosterol is also capable to inhibit the selfassembly of $A \beta$ peptides, entangling with peptides and forming a hydrophobic core with residues Phe-19 and Phe-20. ${ }^{7}$
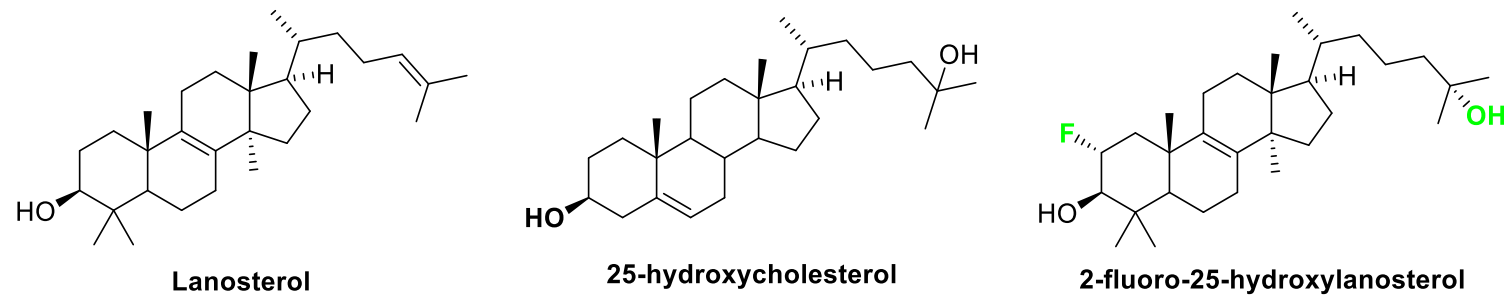

Figure 2. Chemical structures of lanosterol, 25-hydroxycholesterol, and 2-fluoro-25-hydroxylanosterol. 
The improvement of biological properties of natural occurring lanosterol by chemical modification is a critical example of how the right chemical modification in a natural scaffold could lead to highly efficient molecules for therapeutic purposes. Over the years, steroid group compounds, particularly cholesterol, have played a significant interest for organic synthesis, due to good availability, low cost and easily derivatization of the functional groups. Many useful chemical and enzymatic reactions are now widely used for multi-step steroid transformations, leading to important products. ${ }^{3,8}$ The chemical transformations range from simple ones, like manipulations of functional groups, to more complex ones such $\mathrm{C}-\mathrm{H}$ activation or $\mathrm{C}-\mathrm{C}$ bonds formation with organometallic reagents. ${ }^{8}$ From the literature analysis one can realize that highly substituted pyrans tethered with $-\mathrm{NH}_{2}$ and $-\mathrm{CN}$ functionalities at $\mathrm{C}-2$ and $\mathrm{C}-3$ positions (Figure 3), have important pharmacological properties. ${ }^{9-11}$ Furthermore, it should be highlighted the significance of the 2-amino-3-cyano4-phenylpyran pharmacophoric fragment (highlighted in red) in AChE inhibitors ${ }^{12}$ or even as anticancer ${ }^{13}$ and antibacterial ${ }^{14}$ molecules (Figure 3 ).
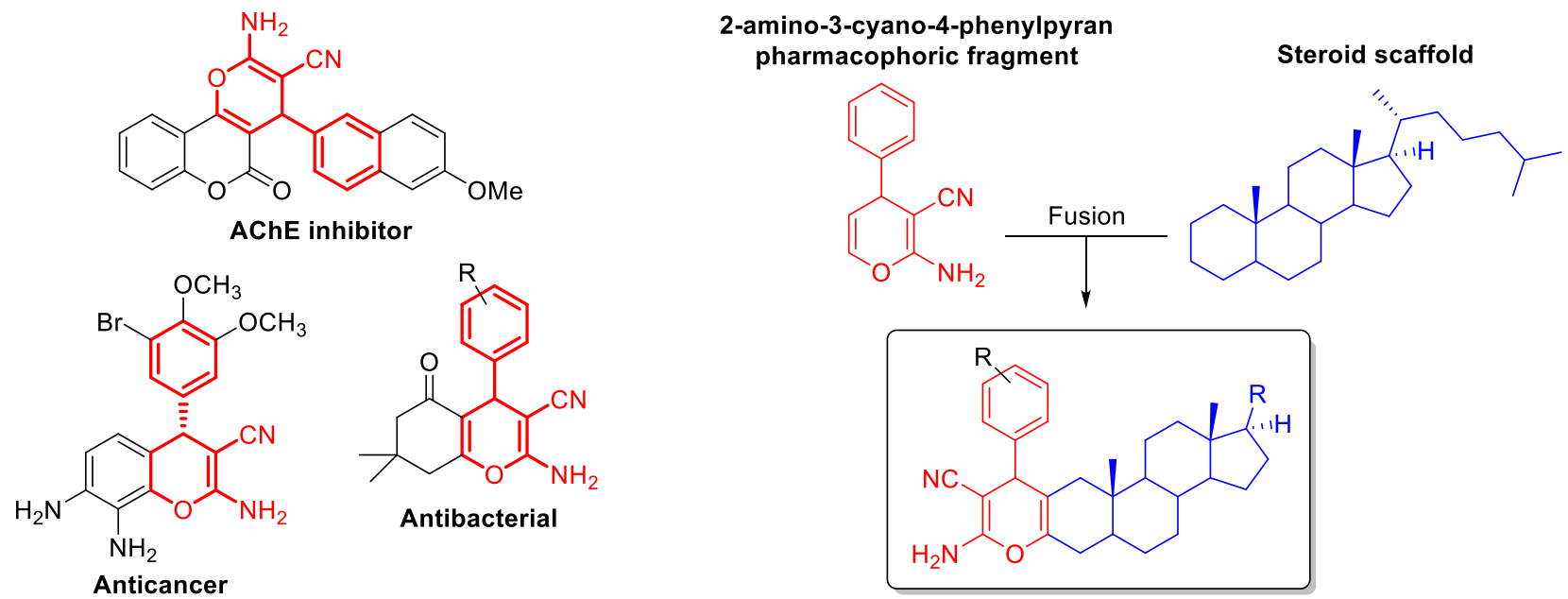

Figure 3. A-ring fused pyran derivatives.

Following our interest in modified steroidal compounds with biological applications, we have looked at the chemical modification of cholestane A-ring, by following a two-step synthetic procedure in order to reach a three-substituted fused pyran heterocyclic ring as depicted in Figure 3. From the retrosynthetic analysis, one can expect that the initial aldol reaction can occur at C-2 or C-4 positions of the steroid scaffold (Figure 4). The selectivity towards one particular position will allow the selective fusion of pyran ring at one specific A-ring side (Figure 4). To the best of our knowledge, herein we present for the first time the A-ring functionalization of cholestane scaffold with highly substituted pyrans tethered with $-\mathrm{NH}_{2}$ and $-\mathrm{CN}$ functionalities (Figure 4).
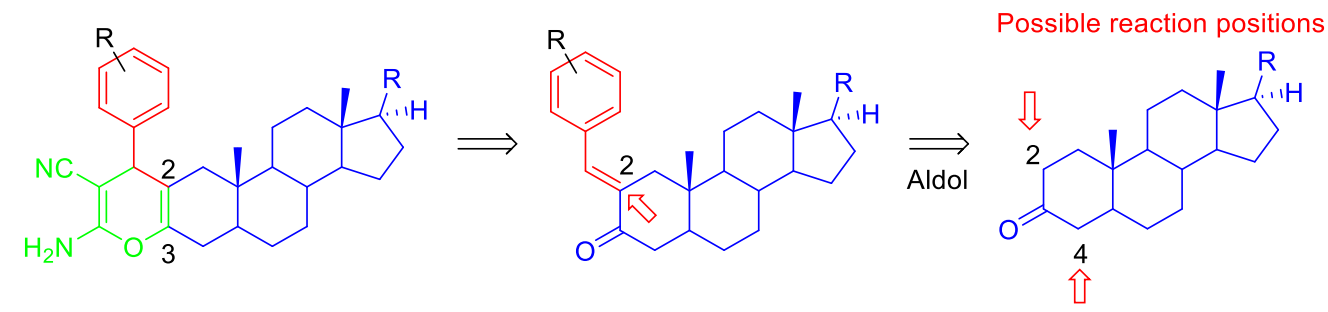

Figure 4. Retrosynthetic analysis of the two-step synthetic methodology. Regioselective functionalization of C2:C-3 A-ring side of cholestane. 


\section{Results and Discussion}

The planned synthetic strategy for the preparation of pyran-fused cholesterol derivatives $4 a-f$ encompasses two reaction steps: 1 ) the aldol reaction of cholestan-3-one 1 and a series of benzaldehydes $2 a-f$ to give the intermediate $\alpha, \beta$-unsaturated carbonyl derivatives 3a-f; and 2) the microwave (MW)-assisted cyclization reaction of $\alpha, \beta$-unsaturated carbonyl derivatives 3a-f and malononitrile (Scheme 1 ). The first reaction step afforded the $\alpha, \beta$-unsaturated carbonyl 3a-f (arbitrary numbering for NMR analysis, Figure 5 ) in good yields (70-80\%), in the presence of $t$-BuOK (5 equiv) as base. The reaction was quite selective towards the $\mathrm{C}-2$ position of the steroid scaffold (see ${ }^{1} \mathrm{H}$ and ${ }^{13} \mathrm{C}$ NMR spectra in SI) (Scheme 1). As we used an excess of benzaldehydes $2 \mathrm{a}-\mathrm{f}$ ( 3 equiv), it was expected to have competition between C-2 and C-4 positions, however, no $\mathrm{C}-4$ reaction products were detected in the reaction media. Possibly this might be due to the more steric hindrance of carbon at position 4 of the steroid scaffold.
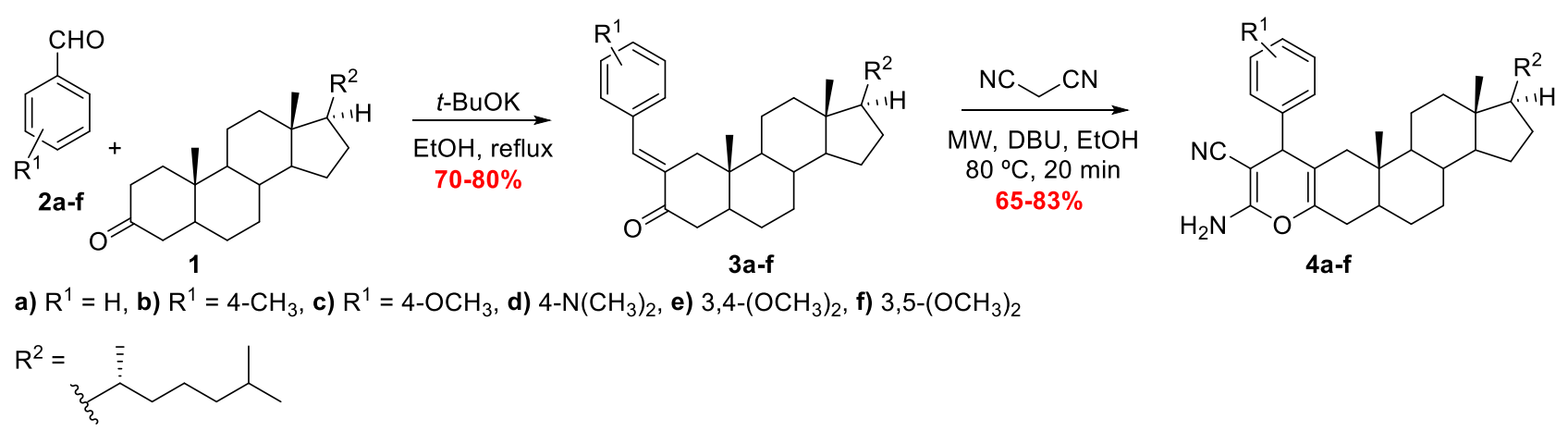

Scheme 1. Synthetic strategy for the two-step preparation of pyran-fused cholesterol derivatives $4 a-f$.

The second step of the synthetic strategy was not straightforward, and optimization of the reaction conditions was required. To do so, we used the $4-\mathrm{CH}_{3} \alpha, \beta$-unsaturated carbonyl derivative $3 \mathbf{b}$ as model substrate and the outcome of the optimization study is summarized in Table 1 . In the first attempts, we evaluated the effect of increasing amounts of malononitrile ( 1 to 5 equiv), ending up with increasing yields (up to $\mathbf{7 5 \%}$ ) of the desired pyran derivative $\mathbf{4 b}$ (mixture of two diastereomers, Figure 5) (Table 1, entries 1-3). When the amount of malononitrile was further increased to 10 and 20 equiv, the formation of 2aminoisophthalonitrile derivative $\mathbf{5 b}$ (blue inset) was observed in 30 and $33 \%$ yields, respectively, with a consequent decrease in the obtained yield of the respective pyran derivative $\mathbf{4 b}$ (Table 1 , entries 4 and 5 ). Changing the substituent from $\mathrm{R}^{1}=\mathrm{CH}_{3}$ to $\mathrm{R}^{1}=\mathrm{OCH}_{3}$ no better improvements were observed and the formation of 2-aminoisophthalonitrile 5 c took place in $30-37 \%$ yields (Table 1 , entries $4-7$ ). Then it can be concluded that the formation of 2-aminoisophthalonitriles $\mathbf{5}$ depends on the amount of malononitrile used in the reaction. The synthesis of 2-aminoisophthalonitriles $\mathbf{5}$, could be explained on the basis of the following mechanism: i) Michael-addition of malononitrile to the $\beta$-position of $\alpha, \beta$-unsaturated carbonyl derivative 3, followed by 1,2-addition of a second molecule of malononitrile and dehydration to give intermediate III (Scheme 2); ii) base-catalyzed intramolecular attack to cyanide group to give intermediate IV, followed by a [1,5] proton shift giving $\mathbf{V}$ (Scheme 2); iii) HCN elimination ending up with the formation of 2aminoisophthalonitriles 5 (Scheme 2).

With the adequate amount of malononitrile well established in order to drive the reaction towards the desired pyran derivatives $\mathbf{4}$, we also struggled to further improve the yield of pyran derivative $\mathbf{4 b}$. Increasing 
amounts of DBU (10 to 30 mol\%) were attempted, as well other base-catalysts such as $\mathrm{Et}_{3} \mathrm{~N}$ and $\mathrm{K}_{2} \mathrm{CO}_{3}(\mathrm{Table}$ 1 , entries 8-11). The isolated yield of pyran $\mathbf{4 b}$ remained unaffected with the increasing amounts of DBU (Table 1, entries 3, 8 and 9), and the switch from DBU to $\mathrm{Et}_{3} \mathrm{~N}$ and $\mathrm{K}_{2} \mathrm{CO}_{3}$, ended up with a slight decrease in the isolated yield of $\mathbf{4 b}$ (Table 1 , entries 10 and 11 ). The screening of the reaction conditions indicated that the higher possible yield for derivative $\mathbf{4 b} \mathbf{b}(\mathbf{7 5} \%)$ is obtained using 5 equiv of malononitrile, in the presence of 10 mol\% of DBU (Table 1, entry 3). Therefore, these reaction conditions were further applied to the remaining $\alpha, \beta$-unsaturated carbonyl derivatives 3a-f, giving the corresponding pyran derivatives $4 a-f$ in $65-83 \%$ yield (Table 1, entries 3, 12-16).
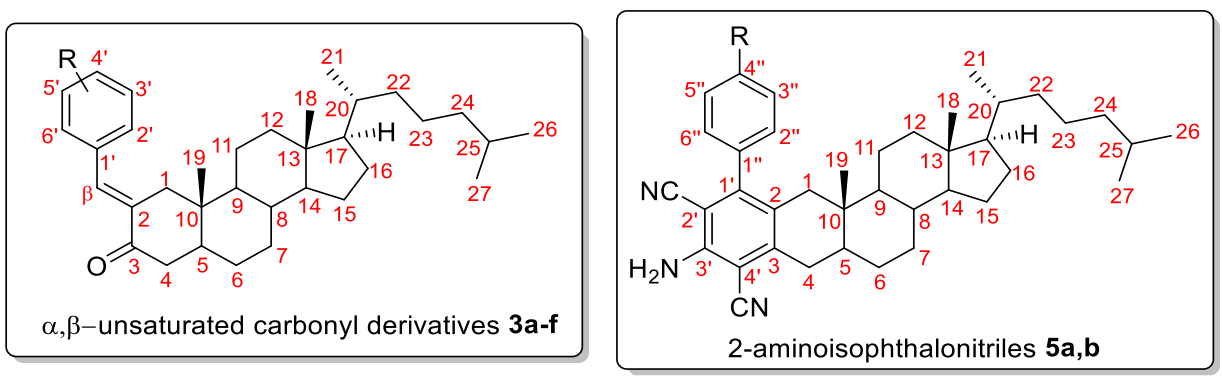

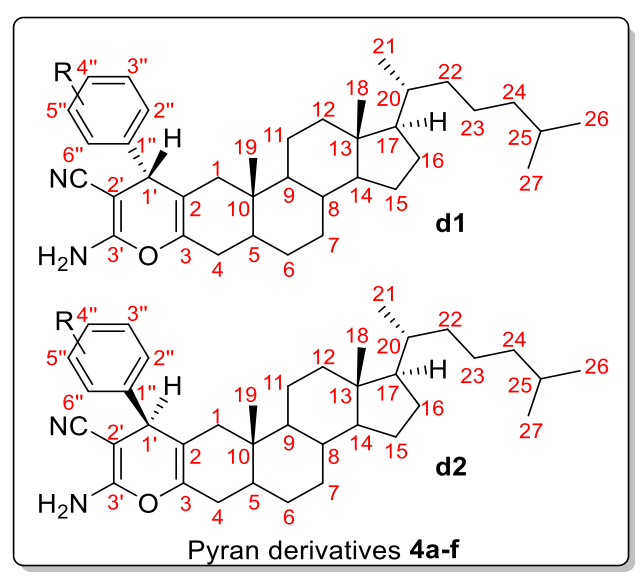

Figure 5. Arbitrary numbering of compounds 3a-f, $\mathbf{4 a - f}$ and $\mathbf{5 a , b}$ for NMR analysis and characterization.

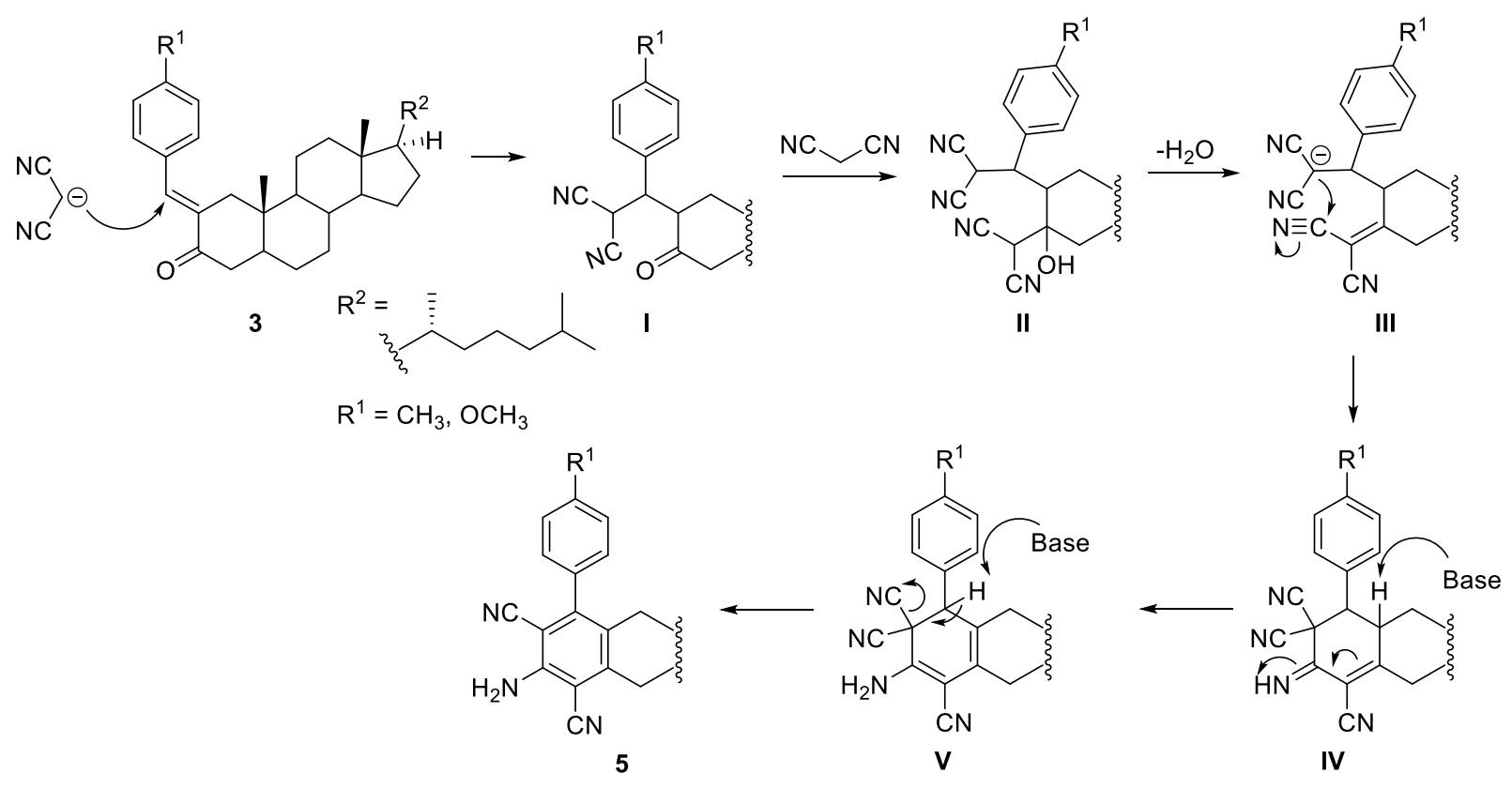

Scheme 2. Plausible mechanism for the formation of 2-aminoisophthalonitrile derivatives $\mathbf{5 b , c}$. 
Table 1. Reaction conditions screening and reaction scope for the synthesis of derivatives $4 a-f$
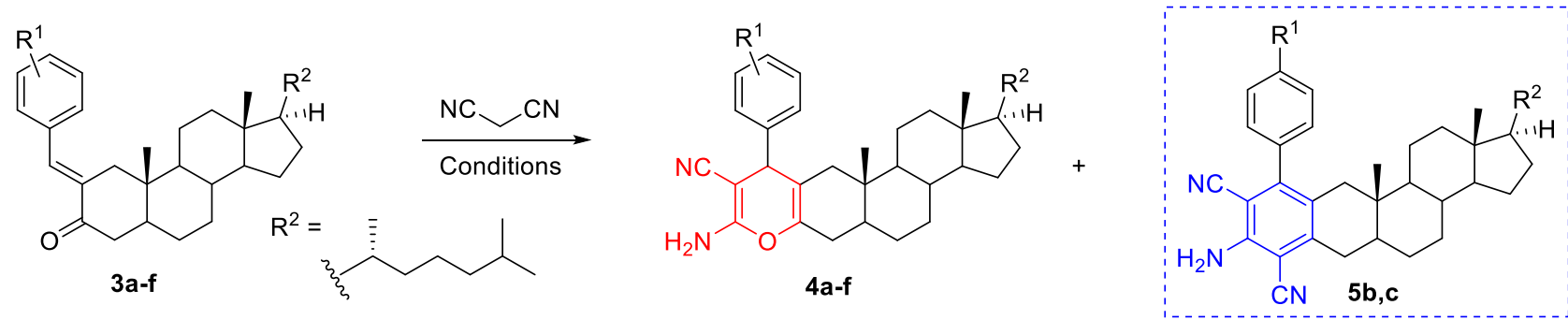

a) $\mathrm{R}_{1}=\mathrm{H} ;$ b) $\mathrm{R}^{1}=4-\mathrm{CH}_{3} ;$ c) $\mathrm{R}^{1}=4-\mathrm{OCH}_{3} ;$ d) $\left.\left.\mathrm{R}^{1}=4-\mathrm{N}\left(\mathrm{CH}_{3}\right)_{2} ; \mathbf{e}\right) \mathrm{R}^{1}=3,4-\left(\mathrm{OCH}_{3}\right)_{2} ; \mathbf{f}\right) \mathrm{R}^{1}=3,5-\left(\mathrm{OCH}_{3}\right)_{2}$

REACTION CONDITIONS SCREENING

\begin{tabular}{|c|c|c|c|c|}
\hline Entry & Derivative (R) & Reaction conditions [a] & 4 Yield (\%) ${ }^{[b]}$ & 5 Yield $(\%)^{[b]}$ \\
\hline 1 & $\mathbf{3 b}\left(4-\mathrm{CH}_{3}\right)$ & Malononitrile ( 1 equiv), DBU (10 mol\%) & 25 & --- \\
\hline 2 & $\mathbf{3 b}\left(4-\mathrm{CH}_{3}\right)$ & Malononitrile ( 3 equiv), DBU (10 mol\%) & 33 & --- \\
\hline 3 & $3 \mathbf{b}\left(4-\mathrm{CH}_{3}\right)$ & Malononitrile (5 equiv), DBU (10 mol\%) & 75 & --- \\
\hline 4 & $\mathbf{3 b}\left(4-\mathrm{CH}_{3}\right)$ & Malononitrile (10 equiv), DBU (10 mol\%) & 50 & 30 \\
\hline 5 & $\mathbf{3 b}\left(4-\mathrm{CH}_{3}\right)$ & Malononitrile (20 equiv), DBU (10 mol\%) & 41 & 33 \\
\hline 6 & $3 \mathrm{c}\left(4-\mathrm{OCH}_{3}\right)$ & Malononitrile (10 equiv), DBU (10 mol\%) & 47 & 37 \\
\hline 7 & $3 \mathrm{c}\left(4-\mathrm{OCH}_{3}\right)$ & Malononitrile (20 equiv), DBU (10 mol\%) & 51 & 35 \\
\hline 8 & $3 \mathbf{b}\left(4-\mathrm{CH}_{3}\right)$ & Malononitrile (5 equiv), DBU (20 mol\%) & 71 & --- \\
\hline 9 & $3 \mathbf{b}\left(4-\mathrm{CH}_{3}\right)$ & Malononitrile (5 equiv), DBU (30 mol\%) & 73 & --- \\
\hline 10 & $3 \mathbf{b}\left(4-\mathrm{CH}_{3}\right)$ & Malononitrile (5 equiv), $\mathrm{Et}_{3} \mathbf{N}$ (10 mol\%) & 61 & --- \\
\hline 11 & $\mathbf{3 b}\left(4-\mathrm{CH}_{3}\right)$ & Malononitrile (5 equiv), $\mathbf{K}_{2} \mathrm{CO}_{3}(10 \mathrm{~mol} \%)$ & 55 & --- \\
\hline \multicolumn{5}{|c|}{ REACTION SCOPE } \\
\hline 12 & $3 a(H)$ & Malononitrile (5 equiv), DBU (10 mol\%) & 78 & --- \\
\hline 13 & $3 c\left(4-\mathrm{OCH}_{3}\right)$ & Malononitrile (5 equiv), DBU (10 mol\%) & 80 & --- \\
\hline 14 & $3 d\left[4-\mathrm{N}\left(\mathrm{CH}_{3}\right)_{2}\right]$ & Malononitrile (5 equiv), DBU (10 mol\%) & 65 & --- \\
\hline 15 & $3 e\left(3,4-\mathrm{OCH}_{3}\right)$ & Malononitrile (5 equiv), DBU (10 mol\%) & 83 & --- \\
\hline 16 & $3 f\left(3,5-\mathrm{OCH}_{3}\right)$ & Malononitrile (5 equiv), DBU (10 mol\%) & 77 & --- \\
\hline
\end{tabular}

[a] Reactions carried out using $0.1 \mathrm{mmol}$ of 3, in EtOH $(2 \mathrm{~mL})$ as solvent, under $\mathrm{MW}$-irradiation at $80{ }^{\circ} \mathrm{C}$ for 20 $\min ;[b]$ isolated yield.

\section{Conclusions}

A series of six pyran-fused cholestane derivatives $4 a-f$ was synthesized in good yields, exploring the reaction of $\alpha, \beta$-unsaturated carbonyl compounds 3a-f with malononitrile, in the presence of DBU. The initial aldol reaction step of cholestan-3-one with benzaldehydes was regioselective towards C-2 position, which is the less hindered position of the steroid scaffold. The two-step synthetic methodology, rather than the one-pot procedure, takes advantage of the aldol reaction regioselectivity, granting the selective fusion of the threesubstituted pyran ring at the C2-C3 positions of cholestane A-ring. In the presence of a high excess of malononitrile, there is the formation of 2-aminoisophthalonitrile fused to C2-C3 of the cholestane A-ring. This is a competing side reaction when higher amounts of malononitrile are input into the reaction. 


\section{Experimental Section}

General. All chemicals were commercially available except those having the synthesis described. All reaction mixtures were monitored by thin layer chromatography (TLC) using commercial TLC plates (Merck Kieselgel 60 F254). The plates were observed under UV light at 254 and $365 \mathrm{~nm}$. Melting points were determined using a Reichert Thermovar apparatus fitted with a microscope and are uncorrected. NMR spectra were recorded in Bruker Avance 300 spectrometer (300 for ${ }^{1} \mathrm{H}$ and $75 \mathrm{MHz}$ for ${ }^{13} \mathrm{C}$ ), in $\mathrm{CDCl}_{3}$ as solvent, if not stated otherwise. Chemical shifts $(\delta)$ are reported in ppm and coupling constants $(J)$ in $\mathrm{Hz}$; internal standard was residual peak of the solvent. Unequivocal ${ }^{13} \mathrm{C}$ assignments were made with the aid of $2 \mathrm{D} g \mathrm{HSQC}$ and $g \mathrm{HMBC}$ (delays for onebond and long-range $J \mathrm{C} / \mathrm{H}$ couplings were optimised for 145 and $7 \mathrm{~Hz}$, respectively) experiments. Highresolution mass spectra analysis (HRMS-ESI) were performed on a microTOF (focus) mass spectrometer. lons were generated using an Apolloll (ESI) source. Ionization was achieved by electrospray, using a voltage of 4500 $\mathrm{V}$ applied to the needle, and a counter voltage between 100 and $150 \mathrm{~V}$ applied to the capillary.

General procedure for the synthesis of $\boldsymbol{\alpha}, \boldsymbol{\beta}$-unsaturated carbonyl compounds (3a-f). To a stirring solution of cholestan-3-one 1 (200 mg, $0.5 \mathrm{mmol}$ ) and $t$-BuOK $(290 \mathrm{mg}, 2.5 \mathrm{mmol})$ in refluxing EtOH $(5 \mathrm{~mL})$, was added the appropriate benzaldehyde 2 a-f $(1.5 \mathrm{mmol})$. The resulting reaction mixture was kept under reflux for $2-3 \mathrm{~h}$. After completion of the reaction (TLC) the mixture was poured into cold water, the $\mathrm{pH}$ adjusted to 4 with $10 \%$ aqueous $\mathrm{HCl}$ and the resulting precipitate recovered by filtration. The crude precipitate was dissolved in $\mathrm{CH}_{2} \mathrm{Cl}_{2}$ $(10 \mathrm{~mL})$, dried over anhydrous $\mathrm{Na}_{2} \mathrm{SO}_{4}$ and concentrated under reduced pressure. The crude product was then purified by preparative TLC using hexane:ethyl acetate (10:1) as eluent.

(5S,8R,9S,10S,13R,14S,17R)-2-[(E)-Benzylidene]-10,13-dimethyl-17-[(R)-6-methylheptan-2-yl]hexadecahydro-3H-cyclopenta[a]phenanthren-3-one (3a). (172 mg, 70\%), mp 97-99 ${ }^{\circ} \mathrm{C} .{ }^{1} \mathrm{H}-\mathrm{NMR}\left(300 \mathrm{MHz}, \mathrm{CDCl}_{3}\right): \delta=$ $0.65\left(\mathrm{~s}, 3 \mathrm{H}, 18\right.$ - or $\left.19-\mathrm{CH}_{3}\right), 0.79\left(\mathrm{~s}, 3 \mathrm{H}, 18\right.$ - or $\left.19-\mathrm{CH}_{3}\right), 0.85-2.29(\mathrm{~m}, 36 \mathrm{H}), 2.45$ (dd, 1H, H-4, J 18.6, 5.4 Hz), $3.11(\mathrm{~d}, 1 \mathrm{H}, \mathrm{H}-1, \mathrm{~J} 15.8 \mathrm{~Hz}), 7.31-7.41\left(\mathrm{~m}, 5 \mathrm{H}, \mathrm{H}-2^{\prime}, 3^{\prime}, 4^{\prime}, 5^{\prime}, 6^{\prime}\right), 7.55-7.56(\mathrm{~m}, 1 \mathrm{H}, \mathrm{H}-\beta)$ ppm. ${ }^{13} \mathrm{C}-\mathrm{NMR}(75 \mathrm{MHz}$, $\left.\mathrm{CDCl}_{3}\right): \delta=11.9,12.0,18.7,21.4,22.6,22.8,23.8,24.2,28.0,28.2,28.7,31.5,35.4,35.8,35.9,36.1,39.5,39.9$, 41.8, 42.4, 42.5, 42.9, 53.6, 56.3, 56.4, 128.4 (C-2',6'), 128.5 (C-4'), 130.3 (C-3', 5'), 135.4 (C-1'), 135.7 (C-2), 137.1 (C- $\beta$ ), 201.8 (C-3) ppm. HRMS (ESI $\left.{ }^{+}\right): m / z[M+H]^{+}$calcd for $\mathrm{C}_{34} \mathrm{H}_{51} \mathrm{O}: 475.3934$; found 475.3960.

(5S,8R,9S,10S,13R,14S,17R)-10,13-Dimethyl-2-[(E)-4-methylbenzylidene]-17-[(R)-6-methylheptan-2-yl]hexadecahydro-3H-cyclopenta[a]phenanthren-3-one (3b). (202 mg, 80\%), mp 149-151 ${ }^{\circ} \mathrm{C} .{ }^{1} \mathrm{H}-\mathrm{NMR}(300 \mathrm{MHz}$, $\left.\mathrm{CDCl}_{3}\right): \delta=0.65\left(\mathrm{~s}, 3 \mathrm{H}, 18\right.$ - or $\left.19-\mathrm{CH}_{3}\right), 0.78\left(\mathrm{~s}, 3 \mathrm{H}, 18\right.$ - or $\left.19-\mathrm{CH}_{3}\right), 0.85-2.27(\mathrm{~m}, 36 \mathrm{H}), 2.37\left(\mathrm{~s}, 3 \mathrm{H}, 4^{\prime}-\mathrm{CH}_{3}\right), 2.44$ (dd, $1 \mathrm{H}, \mathrm{H}-4, J$ 18.7, $5.4 \mathrm{~Hz}), 3.10(\mathrm{~d}, 1 \mathrm{H}, \mathrm{H}-1, J 15.8 \mathrm{~Hz}), 7.20$ (d, 2H, H-3', 5', J $8.1 \mathrm{~Hz}), 7.30$ (d, $2 \mathrm{H}, \mathrm{H}-2^{\prime}, 6^{\prime}, J 8.1$

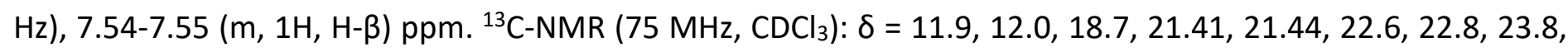
24.2, 28.0, 28.2, 28.7, 31.5, 35.4, 35.8, 35.9, 36.2, 39.5, 39.9, 42.0, 42.3, 42.5, 42.8, 53.6, 56.3, 56.4, 129.1 (C$\left.3^{\prime}, 5^{\prime}\right), 130.5\left(C^{\prime}-2^{\prime}, 6^{\prime}\right), 132.9\left(C-1^{\prime}\right), 134.6(C-2), 137.3(C-\beta), 138.8$ (C-4'), 201.7 (C-3) ppm. HRMS (ESI $): m / z$ $[\mathrm{M}+\mathrm{H}]^{+}$calcd for $\mathrm{C}_{35} \mathrm{H}_{53} \mathrm{O}: 489.4091$; found 489.4098 .

(5S,8R,9S,10S,13R,14S,17R)-2-[(E)-4-Methoxybenzylidene]-10,13-dimethyl-17-[(R)-6-methylheptan-2-yl]hexadecahydro-3H-cyclopenta[a]phenanthren-3-one (3c). (209 mg, 80\%), mp 74-76 ${ }^{\circ} \mathrm{C} .{ }^{1} \mathrm{H}-\mathrm{NMR}(300 \mathrm{MHz}$, $\left.\mathrm{CDCl}_{3}\right): \delta=0.65\left(\mathrm{~s}, 3 \mathrm{H}, 18\right.$ - or $\left.19-\mathrm{CH}_{3}\right), 0.79\left(\mathrm{~s}, 3 \mathrm{H}, 18\right.$ - or $\left.19-\mathrm{CH}_{3}\right), 0.85-2.27(\mathrm{~m}, 36 \mathrm{H}), 2.43(\mathrm{dd}, 1 \mathrm{H}, \mathrm{H}-4, J 18.7$, $5.4 \mathrm{~Hz}$ ), $3.08(\mathrm{~d}, 1 \mathrm{H}, \mathrm{H}-1, J 16.1 \mathrm{~Hz}), 3.84\left(\mathrm{~s}, 3 \mathrm{H}, 4^{\prime}-\mathrm{OCH}_{3}\right), 6.93\left(\mathrm{~d}, 2 \mathrm{H}, \mathrm{H}-3^{\prime}, 5^{\prime}, J 8.8 \mathrm{~Hz}\right), 7.38\left(\mathrm{~d}, 2 \mathrm{H}, \mathrm{H}-2^{\prime}, 6^{\prime}, J\right.$ $8.8 \mathrm{~Hz}), 7.54-7.55(\mathrm{~m}, 1 \mathrm{H}, \mathrm{H}-\beta)$ ppm. ${ }^{13} \mathrm{C}-\mathrm{NMR}\left(75 \mathrm{MHz}, \mathrm{CDCl}_{3}\right): \delta=11.9,12.0,18.7,21.5,22.6,22.8,23.8,24.2$, 28.0, 28.2, 28.7, 31.5, 35.4, 35.8, 36.2, 39.5, 40.0, 42.1, 42.5, 42.7, 53.7, 55.3, 56.3, 56.4, 113.9 (C-3', 5'), 128.3 $\left(\mathrm{C}-1^{\prime}\right), 132.3\left(\mathrm{C}-2^{\prime}, 6^{\prime}\right), 133.3$ (C-2), 137.2 (C- $\beta$ ), 159.9 (C-4'), 201.5 (C-3) ppm. HRMS (ESI $): m / z[M+H]^{+}$calcd for $\mathrm{C}_{35} \mathrm{H}_{53} \mathrm{O}_{2}$ : 505.4040; found 505.4040. 
(5S,8R,9S,10S,13R,14S,17R)-2-[(E)-4-(Dimethylamino)benzylidene]-10,13-dimethyl-17-[(R)-6-methylheptan2-yl]hexadecahydro-3H-cyclopenta[a]phenanthren-3-one (3d). (187 mg, 70\%), mp 167-168 ${ }^{\circ} \mathrm{C} .{ }^{1} \mathrm{H}-\mathrm{NMR}(300$ $\left.\mathrm{MHz}, \mathrm{CDCl}_{3}\right): \delta=0.66\left(\mathrm{~s}, 3 \mathrm{H}, 18\right.$ - or $\left.19-\mathrm{CH}_{3}\right), 0.79\left(\mathrm{~s}, 3 \mathrm{H}, 18\right.$ - or 19- $\left.\mathrm{CH}_{3}\right), 0.86-2.27(\mathrm{~m}, 36 \mathrm{H}), 2.41(\mathrm{dd}, 1 \mathrm{H}, \mathrm{H}-4, J$ 18.9, $5.5 \mathrm{~Hz}$ ), 3.02 [s, 6H, 4'-N( $\left.\left.\mathrm{CH}_{3}\right)_{2}\right], 3.09$ (d, 1H, H-1, J $\left.15.7 \mathrm{~Hz}\right), 6.71\left(\mathrm{~d}, 2 \mathrm{H}, \mathrm{H}-3^{\prime}, 5^{\prime}, J 8.7 \mathrm{~Hz}\right), 7.40(\mathrm{~d}, 2 \mathrm{H}, \mathrm{H}-$ $2^{\prime}, 6^{\prime}$, J $\left.8.7 \mathrm{~Hz}\right), 7.58-7.59(\mathrm{~m}, 1 \mathrm{H}, \mathrm{H}-\beta)$ ppm. ${ }^{13} \mathrm{C}-\mathrm{NMR}\left(75 \mathrm{MHz}, \mathrm{CDCl}_{3}\right): \delta=12.0,18.7,21.5,22.6,22.8,23.9$, 24.3, 28.0, 28.3, 28.7, 31.6, 35.4, 35.6, 35.8, 36.2, 39.5, 40.0, 40.1, 41.9, 42.5, 53.8, 56.3, 56.4, 111.6 (C-3',5'), $123.6\left(\mathrm{C}-1^{\prime}\right), 130.5(\mathrm{C}-2), 132.7\left(\mathrm{C}-2^{\prime}, 6^{\prime}\right), 138.5(\mathrm{C}-\beta), 150.5\left(\mathrm{C}-4^{\prime}\right), 201.1(\mathrm{C}-3) \mathrm{ppm} . \mathrm{HRMS}\left(\mathrm{ESI} \mathrm{I}^{+}\right): \mathrm{m} / \mathrm{z}[\mathrm{M}+\mathrm{H}]^{+}$ calcd for $\mathrm{C}_{36} \mathrm{H}_{56} \mathrm{ON}$ : 518.4356; found 518.4357 .

(5S,8R,9S,10S,13R,14S,17R)-2-[(E)-3,4-Dimethoxybenzylidene]-10,13-dimethyl-17-[(R)-6-methylheptan-2yl]hexadecahydro-3H-cyclopenta[a]phenanthren-3-one (3e). (207 mg, 75\%), mp 58-61 ${ }^{\circ} \mathrm{C} .{ }^{1} \mathrm{H}-\mathrm{NMR}(300 \mathrm{MHz}$, $\left.\mathrm{CDCl}_{3}\right): \delta=0.66\left(\mathrm{~s}, 3 \mathrm{H}, 18\right.$ - or $\left.19-\mathrm{CH}_{3}\right), 0.80\left(\mathrm{~s}, 3 \mathrm{H}, 18\right.$ - or $\left.19-\mathrm{CH}_{3}\right), 0.85-2.28(\mathrm{~m}, 36 \mathrm{H}), 2.44(\mathrm{dd}, 1 \mathrm{H}, \mathrm{H}-4, J 18.8$, $5.4 \mathrm{~Hz}), 3.13(\mathrm{~d}, 1 \mathrm{H}, \mathrm{H}-1, \mathrm{~J} 15.8 \mathrm{~Hz}), 3.89\left(\mathrm{~s}, 3 \mathrm{H}, 3^{\prime}\right.$ - or 4'-OCH $\left.4^{\prime}\right), 3.92\left(\mathrm{~s}, 3 \mathrm{H}, 3^{\prime}-\right.$ or 4'-OCH $\left.4^{\prime}\right), 6.90\left(\mathrm{~d}, 1 \mathrm{H}, \mathrm{H}-5^{\prime}, J\right.$ $8.4 \mathrm{~Hz}$ ), $6.96\left(\mathrm{~d}, 1 \mathrm{H}, \mathrm{H}-2^{\prime}\right.$, J $\left.2.0 \mathrm{~Hz}\right), 7.05$ (dd, 1H, H-6', J 8.4, 2.0 Hz), 7.53-7.54 (m, 1H, H- $\beta$ ) ppm. ${ }^{13} \mathrm{C}-\mathrm{NMR}(75$ $\left.\mathrm{MHz}_{2} \mathrm{CDCl}_{3}\right): \delta=12.0,18.7,21.5,22.6,22.8,23.8,24.2,28.0,28.2,28.7,31.5,35.4,35.8,36.1,39.5,39.9,42.1$, $42.2,42.5,42.7,53.7,55.85,55.90,56.29,56.35,110.8$ (C-5'), $113.7\left(C-2^{\prime}\right), 123.9$ (C-6'), 128.6 (C-1'), 133.5 (C-

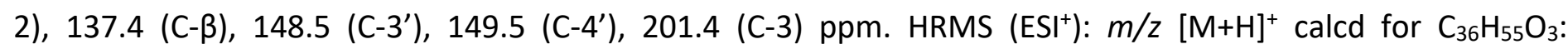
535.4146; found 535.4160 .

(5S,8R,9S,10S,13R,14S,17R)-2-[(E)-3,5-Dimethoxybenzylidene]-10,13-dimethyl-17-[(R)-6-methylheptan-2yl]hexadecahydro-3H-cyclopenta[a]phenanthren-3-one (3f). (213 mg, 77\%), mp 62-65 ${ }^{\circ} \mathrm{C} .{ }^{1} \mathrm{H}-\mathrm{NMR}(300 \mathrm{MHz}$, $\left.\mathrm{CDCl}_{3}\right): \delta=0.65\left(\mathrm{~s}, 3 \mathrm{H}, 18\right.$ - or $\left.19-\mathrm{CH}_{3}\right), 0.80\left(\mathrm{~s}, 3 \mathrm{H}, 18\right.$ - or $\left.19-\mathrm{CH}_{3}\right), 0.85-2.28(\mathrm{~m}, 36 \mathrm{H}), 2.44(\mathrm{dd}, 1 \mathrm{H}, \mathrm{H}-4, J 18.6$, $5.3 \mathrm{~Hz}), 3.13(\mathrm{~d}, 1 \mathrm{H}, \mathrm{H}-1, J 15.8 \mathrm{~Hz}), 3.80\left(\mathrm{~s}, 6 \mathrm{H}, 3^{\prime}-\right.$ and $\left.^{\prime}-\mathrm{OCH}_{3}\right), 6.44\left(\mathrm{t}, 1 \mathrm{H}, \mathrm{H}-4^{\prime}, J 2.2 \mathrm{~Hz}\right), 6.52\left(\mathrm{~d}, 2 \mathrm{H}, \mathrm{H}-2^{\prime}, 6^{\prime}\right.$, J $2.2 \mathrm{~Hz}$ ), 7.46-7.47 (m, 1H, H- $\beta$ ) ppm. ${ }^{13} \mathrm{C}-\mathrm{NMR}\left(75 \mathrm{MHz}, \mathrm{CDCl}_{3}\right): \delta=11.9,12.0,18.7,21.5,22.6,22.8,23.8$, 24.2, 28.0, 28.2, 28.7, 31.5, 35.4, 35.8, 35.9, 36.1, 39.5, 39.9, 41.9, 42.4, 42.5, 42.9, 53.5, 55.4 (3'- and 5'$\left.\mathrm{OCH}_{3}\right), 56.28,56.31,100.7\left(C^{\prime}-4^{\prime}\right), 108.2\left(C^{\prime}-2^{\prime}, 6^{\prime}\right), 135.9(C-2), 137.0(C-\beta), 137.5\left(C-1^{\prime}\right), 160.5\left(C-3^{\prime}, 5^{\prime}\right), 201.7(C-$ 3) ppm. HRMS $\left(\mathrm{ESI}^{+}\right): \mathrm{m} / z[\mathrm{M}+\mathrm{H}]^{+}$calcd for $\mathrm{C}_{36} \mathrm{H}_{55} \mathrm{O}_{3}: 535.4146$; found 535.4155 .

General procedure for the synthesis of pyran-fused compounds (4a-f). The appropriate $\alpha, \beta$-unsaturated carbonyl compound 3a-f $(0.1 \mathrm{mmol})$, malononitrile $(33 \mathrm{mg}, 0.5 \mathrm{mmol})$, DBU $(10 \mathrm{~mol} \%)$ and EtOH $(2 \mathrm{~mL}) \mathrm{were}$ mixed in a CEM Microwave tube. The resulting mixture was heated at $80{ }^{\circ} \mathrm{C}$ for 20 min under MW-irradiation. After that period, the solvent was removed under reduced pressure, the crude product dissolved in $\mathrm{CH}_{2} \mathrm{Cl}_{2}(5$ $\mathrm{mL}$ ) and purified by preparative TLC, using a 2:1 mixture of hexane:ethyl acetate as eluent. The compounds were obtained as a mixture of two diastereomers, arbitrary assigned as $\mathbf{d} \mathbf{1}$ and $\mathbf{d} \mathbf{2}$, from the analysis of ${ }^{1} \mathrm{H},{ }^{13} \mathrm{C}$ NMR, HSQC and HMBC spectra.

Pyran compound (4a) (mixture of $\mathrm{d} 1$ and $\mathrm{d} 2$ diastereomers) $=(1 R, 3 \mathrm{a} S, 3 \mathrm{~b} R, 5 \mathrm{a} S, 10 R / S, 11 \mathrm{aS}, 11 \mathrm{bS}, 13 \mathrm{a} R)-8-$ amino-11a,13a-dimethyl-1-[(R)-6-methylheptan-2-yl]-10-phenyl-1,2,3,3a,3b,4,5,5a,6,10,11,11a,11b,12,13, 13a-hexadecahydrocyclopenta[5,6]naphtho[1,2-g]chromene-9-carbonitrile. (42 mg, 78\%). ${ }^{1} \mathrm{H}-\mathrm{NMR}$ (300 $\left.\mathrm{MHz}_{2} \mathrm{CDCl}_{3}\right): \delta=0.55-2.08(\mathrm{~m}, 88 \mathrm{H}, \mathrm{d} 1+\mathrm{d} 2), 3.75\left(\mathrm{~s}, 2 \mathrm{H}, \mathrm{H}-1^{\prime}, \mathrm{d} 1+\mathrm{d} 2\right), 4.33\left(\mathrm{~s}, 2 \mathrm{H}, 3^{\prime}-\mathrm{NH}_{2}, \mathrm{~d} 2\right), 4.34\left(\mathrm{~s}, 2 \mathrm{H}, 3^{\prime}-\right.$ $\left.\mathrm{NH}_{2}, \mathrm{~d} 1\right), 7.14-7.35$ (m, 10H, H-2", 3", $\left.4^{\prime \prime}, 5^{\prime \prime}, 6^{\prime \prime}, \mathrm{d} 1+\mathrm{d} 2\right)$ ppm. ${ }^{13} \mathrm{C}-\mathrm{NMR}\left(75 \mathrm{MHz}, \mathrm{CDCl}_{3}\right): \delta=11.5,11.9,11.96$, $11.98,18.6,21.1,21.6,22.7,22.8,23.8,23.9,24.2,28.0,28.2,30.4,30.5,31.5,35.0,35.3,35.36,35.40,35.7$, $35.8,36.1,39.5,39.8,39.9,41.5,41.6,42.0,42.1,42.4,43.5,53.2,53.5,56.2,60.9,108.1$ (C-2, d1), 108.5 (C-2, d2), 120.2 (CN, d1), 120.3 (CN, d2), 127.1 (C-4", d1+d2), 127.8 (C-2", 6", d1), 127.9 (C-2", 6", d2), 128.5 (C-

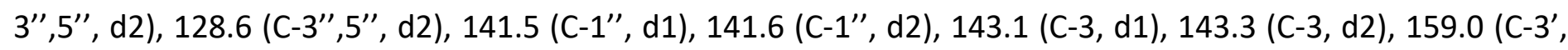
$\mathrm{d} 1+\mathrm{d} 2)$ ppm. HRMS $\left(\mathrm{ESI}^{+}\right): \mathrm{m} / z[\mathrm{M}+\mathrm{H}]^{+}$calcd for $\mathrm{C}_{37} \mathrm{H}_{53} \mathrm{~N}_{2} \mathrm{O}: 541.4152$; found 541.4153. 
Pyran compound (4b) (mixture of $\mathrm{d} 1$ and $\mathrm{d} 2$ diastereomers) $=(1 R, 3 \mathrm{a} S, 3 \mathrm{~b} R, 5 \mathrm{a} S, 10 R / S, 11 \mathrm{aS}, 11 \mathrm{bS}, 13 \mathrm{a} R)-8-$ amino-11a,13a-dimethyl-1-[(R)-6-methylheptan-2-yl]-10-(4-methylphenyl)-

1,2,3,3a,3b,4,5,5a,6,10,11,11a,11b,12,13,13a-hexadecahydrocyclopenta[5,6]naphtho[1,2-g]chromene-9-

carbonitrile. (41 mg, 75\%). ${ }^{1} \mathrm{H}-\mathrm{NMR}\left(300 \mathrm{MHz}_{\mathrm{CDCl}}\right): \delta=0.57-2.09(\mathrm{~m}, 88 \mathrm{H}, \mathrm{d} 1+\mathrm{d} 2), 2.32\left(\mathrm{~s}, 3 \mathrm{H}, 4^{\prime \prime}-\mathrm{CH}_{3}, \mathrm{~d} 2\right)$, $2.33\left(\mathrm{~s}, 3 \mathrm{H}, 4^{\prime \prime}-\mathrm{CH}_{3}, \mathrm{~d} 1\right), 3.71\left(\mathrm{~s}, 2 \mathrm{H}, \mathrm{H}-1^{\prime}\right.$, d1+d2), 4.30 (s, 2H, 3'-NH2, d2), 4.32 (s, 2H, 3'-NH2, d1), 7.02-7.14 (m, 8H, H-2", $3^{\prime \prime}, 5^{\prime \prime}, 6^{\prime \prime}, \mathrm{d} 1+\mathrm{d} 2$ ) ppm. ${ }^{13} \mathrm{C}-\mathrm{NMR}\left(75 \mathrm{MHz}, \mathrm{CDCl}_{3}\right): \delta=11.6,11.91,11.94,12.0,18.6,21.1\left(4^{\prime \prime}-\mathrm{CH}_{3}\right.$, d1), $21.2\left(4^{\prime \prime}-\mathrm{CH}_{3}, \mathrm{~d} 2\right), 22.6,22.8,23.80 .23 .84,24.2,28.0,28.2,30.47,30.53,31.47,31.50,35.0,35.34,35.36$, $35.40,35.75,35.76,36.1,39.5,39.75,39.77,39.9,41.56,41.63,42.0,42.4,43.0,53.2,53.5,56.18,56.24,61.2$, 61.3, 108.3 (C-2, d1), 108.6 (C-2, d2), 120.2 (CN, d1), 120.3 (CN, d2), 127.6 (C-2",6", d1), 127.8 (C-2" ,6", d2),

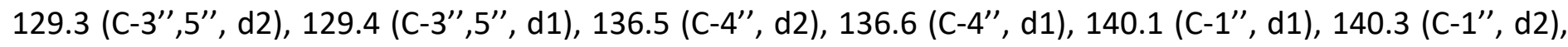
$141.4(\mathrm{C}-3, \mathrm{~d} 1), 141.5$ (C-3, d2), 158.76 (C-3', d2), $158.82\left(\mathrm{C}-3^{\prime}, \mathrm{d} 1\right)$ ppm. HRMS (ESI $\left.{ }^{+}\right): \mathrm{m} / \mathrm{z}[\mathrm{M}+\mathrm{H}]^{+}$calcd for $\mathrm{C}_{38} \mathrm{H}_{55} \mathrm{~N}_{2} \mathrm{O}: 555.4309 ;$ found 555.4323.

Pyran compound (4c) (mixture of $\mathrm{d} 1$ and $\mathrm{d} 2$ diastereomers) $=(1 R, 3 \mathrm{a} S, 3 \mathrm{~b} R, 5 \mathrm{a} S, 10 R / S, 11 \mathrm{aS}, 11 \mathrm{bS}, 13 \mathrm{a} R)-8-$ amino-10-(4-methoxyphenyl)-11a,13a-dimethyl-1-[(R)-6-methylheptan-2-yl]-

1,2,3,3a,3b,4,5,5a,6,10,11,11a,11b,12,13,13a-hexadecahydrocyclopenta[5,6]naphtho[1,2-g]chromene-9-

carbonitrile. (46 mg, 80\%). ${ }^{1} \mathrm{H}-\mathrm{NMR}\left(300 \mathrm{MHz}, \mathrm{CDCl}_{3}\right): \delta=0.56-2.08(\mathrm{~m}, 88 \mathrm{H}, \mathrm{d} 1+\mathrm{d} 2), 3.71\left(\mathrm{~s}, 2 \mathrm{H}, \mathrm{H}-1^{\prime}, \mathrm{d} 1+\mathrm{d} 2\right)$, $3.80\left(\mathrm{~s}, 6 \mathrm{H}, 4^{\prime \prime}-\mathrm{OCH}_{3}, \mathrm{~d} 1+\mathrm{d} 2\right), 4.30\left(\mathrm{~s}, 2 \mathrm{H}, 3^{\prime}-\mathrm{NH}_{2}, \mathrm{~d} 2\right), 4.32\left(\mathrm{~s}, 2 \mathrm{H}, 3^{\prime}-\mathrm{NH}_{2}, \mathrm{~d} 1\right), 6.83\left(\mathrm{~d}, 2 \mathrm{H}, \mathrm{H}-3^{\prime \prime}, 5^{\prime \prime}, J 8.7 \mathrm{~Hz}\right.$,

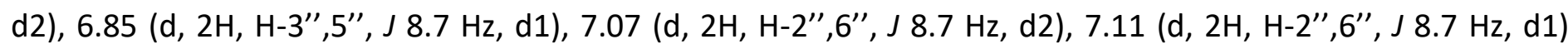
ppm. ${ }^{13} \mathrm{C}-\mathrm{NMR}\left(75 \mathrm{MHz}, \mathrm{CDCl}_{3}\right): \delta=11.6,11.91,11.94,12.0,18.6,21.1,22.6,22.8,23.80,23.84,28.0,28.2$, $30.5,31.5,35.0,35.3,35.4,35.8,36.1,39.5,39.8,41.3,41.6,42.0,42.4,42.6,53.2,53.4,55.21,55.24,56.19$, 56.25, 61.29, 61.32, 108.4 (C-2, d1), 108.7 (C-2, d2), 113.9 (C-3"',5”, d2), 114.0 (C-3"',5", d1), 120.2 (CN, d1), 120.4 (CN, d2), 128.7 (C-2", 6", d1), 128.9 (C-2", 6", d2), 135.3 (C-1", d1), 135.5 (C-1"', d2), 141.3 (C-3, d1), 141.4 (C-3, d2), 158.57 (C-4"', d2), 158.63 (C-4', d1), 158.7 (C-3', d2), 158.8 (C-3', d1) ppm. HRMS (ESI $\left.{ }^{+}\right): m / z$ $[\mathrm{M}+\mathrm{H}]^{+}$calcd for $\mathrm{C}_{38} \mathrm{H}_{55} \mathrm{~N}_{2} \mathrm{O}_{2}: 571.4258$; found 571.4276 .

Pyran compound (4d) (mixture of $\mathrm{d} 1$ and $\mathrm{d} 2$ diastereomers) $=(1 R, 3 \mathrm{a} S, 3 \mathrm{~b} R, 5 \mathrm{a} S, 10 R / S, 11 \mathrm{aS}, 11 \mathrm{bS}, 13 \mathrm{a} R)-8-$ amino-10-[4-(dimethylamino)phenyl]-11a,13a-dimethyl-1-[(R)-6-methylheptan-2-yl]-

1,2,3,3a,3b,4,5,5a,6,10,11,11a,11b,12,13,13a-hexadecahydrocyclopenta[5,6]naphtho[1,2-g]chromene-9-

carbonitrile. (38 mg, 65\%). ${ }^{1} \mathrm{H}-\mathrm{NMR}\left(300 \mathrm{MHz}, \mathrm{CDCl}_{3}\right): \delta=0.57-2.07(\mathrm{~m}, 88 \mathrm{H}, \mathrm{d} 1+\mathrm{d} 2), 2.93\left[\mathrm{~s}, 6 \mathrm{H}, 4^{\prime \prime}-\mathrm{N}\left(\mathrm{CH}_{3}\right)_{2}\right.$, d2], $2.94\left[s, 6 \mathrm{H}, 4^{\prime \prime}-\mathrm{N}\left(\mathrm{CH}_{3}\right)_{2}, \mathrm{~d} 1\right], 3.66\left(\mathrm{~s}, 2 \mathrm{H}, \mathrm{H}-1^{\prime}, \mathrm{d} 1+\mathrm{d} 2\right), 4.26\left(\mathrm{~s}, 2 \mathrm{H}, 3^{\prime}-\mathrm{NH}_{2}, \mathrm{~d} 2\right), 4.28\left(\mathrm{~s}, 2 \mathrm{H}, 3^{\prime}-\mathrm{NH} H_{2}, \mathrm{~d} 1\right)$, 6.66-6.71 (m, 4H, H-3" , 5"', d1+d2), 7.01 (d, 2H, H-2", 6", J $8.7 \mathrm{~Hz}, \mathrm{~d} 2), 7.06$ (d, 2H, H-2", 6", J 8.7 Hz, d1) ppm. ${ }^{13} \mathrm{C}-\mathrm{NMR}\left(125 \mathrm{MHz}, \mathrm{CDCl}_{3}\right): \delta=11.6,11.93,11.95,12.0,18.7,21.1,21.3,22.6,22.8,23.8,23.9,24.2,28.0$, 28.20, 28.23, 29.7, 30.5, 30.6, 31.50, 31.54, 35.0, 35.3, 35.4, 35.76, 35.79, 36.1, 39.5, 39.8, 39.9, 40.68, 40.73, 41.0, 41.6, 42.0, 42.4, 53.2, 53.5, 56.19, 56.23, 56.3, 61.7, 61.8, 108.6 (C-2, d1), 109.0 (C-2, d2), 112.7 (C-3" ", 5",

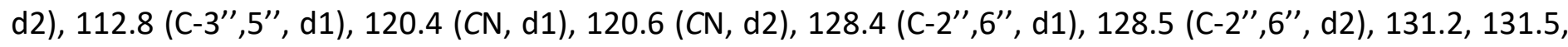
141.1 (C-3, d1), 141.3 (C-3, d2), 149.6 (C-4", $\mathrm{d} 1+\mathrm{d} 2), 158.6$ (C-3', d2), 158.7 (C-3', d1) ppm. HRMS (ESI $\left.{ }^{+}\right): m / z$ $[\mathrm{M}+\mathrm{H}]^{+}$calcd for $\mathrm{C}_{39} \mathrm{H}_{58} \mathrm{~N}_{3} \mathrm{O}: 584.4574$; found 584.4583 .

Pyran compound (4e) (mixture of $\mathrm{d} 1$ and $\mathrm{d} 2$ diastereomers) $=(1 R, 3 \mathrm{a} S, 3 \mathrm{~b} R, 5 \mathrm{a} S, 10 R / S, 11 \mathrm{a} S, 11 \mathrm{bS}, 13 \mathrm{a} R)-8$ amino-10-(3,4-dimethoxyphenyl)-11a,13a-dimethyl-1-[(R)-6-methylheptan-2-yl]1,2,3,3a,3b,4,5,5a,6,10,11,11a,11b,12,13,13a-hexadecahydrocyclopenta[5,6]naphtho[1,2-g]chromene-9carbonitrile. (50 mg, 83\%). ${ }^{1} \mathrm{H}-\mathrm{NMR}\left(300 \mathrm{MHz}, \mathrm{CDCl}_{3}\right): \delta=0.58-2.09(\mathrm{~m}, 88 \mathrm{H}, \mathrm{d} 1+\mathrm{d} 2), 3.70\left(\mathrm{~s}, 1 \mathrm{H}, \mathrm{H}-1^{\prime}, \mathrm{d} 1\right)$, $3.73\left(\mathrm{~s}, 1 \mathrm{H}, \mathrm{H}-1^{\prime}, \mathrm{d} 2\right), 3.85,3.87$ and 3.88 (3s, 12H, 3'- and $\left.4^{\prime \prime}-\mathrm{OCH}_{3}, \mathrm{~d} 1+\mathrm{d} 2\right), 4.34\left(\mathrm{~s}, 2 \mathrm{H}, 3^{\prime}-\mathrm{NH}_{2}, \mathrm{~d} 2\right), 4.37$ (s, $\left.2 \mathrm{H}, 3^{\prime}-\mathrm{NH}_{2}, \mathrm{~d} 1\right), 6.65$ (d, 1H, H-2", J $\left.2.0 \mathrm{~Hz}, \mathrm{~d} 2\right), 6.70-6.83\left(\mathrm{~m}, 5 \mathrm{H}, \mathrm{H}-2^{\prime \prime}, \mathrm{H}-5^{\prime \prime}\right.$ and H-6", d1+d2) ppm. ${ }^{13} \mathrm{C}-\mathrm{NMR}$ $\left(75 \mathrm{MHz}, \mathrm{CDCl}_{3}\right): \delta=11.7,11.91,11.94,12.0,18.6,21.1,21.2,22.6,22.8,23.80,23.84,24.2,28.0,28.2,30.48$, $30.54,31.5,35.0,35.3,35.36,35.40,35.7,35.8,36.1,39.5,39.76,39.83,41.58,41.65,41.67,42.0,42.4,43.1$, 
53.2, 53.5, 55.82, 55.84, 55.9, 56.0, 56.2, 61.1 (C-2', d1), 61.2 (C-2', d2), 108.2 (C-2, d1), 108.5 (C-2, d2), 110.7 (C-2" or C-5", d2), 110.9 (C-2" or C-5", d1), 111.0 (C-2" or C-5", d2), 111.2 (C-2" or C-5", d1), 119.9 (C-6", d1), 120.25 (C-6") d2), 120.26 (CN, d1), 120.3 (CN, d2), 135.8 (C-1" , d1), 135.9 (C-1"', d2), 141.4 (C-3, d2), 141.6 (C3, d2), 148.0 (C-3"', d2), 148.1 (C-3"', d1), 149.1 (C-4", d1+d2), 158.7 (C-3', d2), 158.8 (C-3', d1) ppm. HRMS $\left(\mathrm{ESI}^{+}\right): \mathrm{m} / z[\mathrm{M}+\mathrm{H}]^{+}$calcd for $\mathrm{C}_{39} \mathrm{H}_{57} \mathrm{~N}_{2} \mathrm{O}_{3}: 601.4364$; found 601.4375 .

Pyran compound (4f) (mixture of $\mathrm{d} 1$ and $\mathrm{d} 2$ diastereomers) $=(1 R, 3 \mathrm{a} S, 3 \mathrm{~b} R, 5 \mathrm{aS}, 10 R / S, 11 \mathrm{aS}, 11 \mathrm{bS}, 13 \mathrm{a} R)-8-$ amino-10-(3,5-dimethoxyphenyl)-11a,13a-dimethyl-1-[(R)-6-methylheptan-2-yl]-

\section{1,2,3,3a,3b,4,5,5a,6,10,11,11a,11b,12,13,13a-hexadecahydrocyclopenta[5,6]naphtho[1,2-g]chromene-9-}

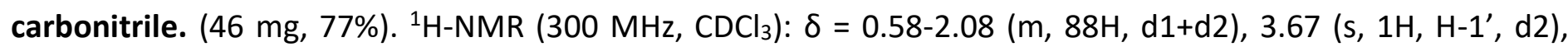
$3.70\left(\mathrm{~s}, 1 \mathrm{H}, \mathrm{H}-1^{\prime}, \mathrm{d} 1\right), 3.77\left(\mathrm{~s}, 6 \mathrm{H}, 3^{\prime \prime}, 5^{\prime \prime}-\mathrm{OCH}_{3}, \mathrm{~d} 2\right), 3.79\left(\mathrm{~s}, 6 \mathrm{H}, 3^{\prime \prime}, 5^{\prime \prime}-\mathrm{OCH}_{3}, \mathrm{~d} 1\right), 4.32\left(\mathrm{~s}, 2 \mathrm{H}, 3^{\prime}-\mathrm{NH}_{2}, \mathrm{~d} 2\right), 4.33(\mathrm{~s}$, $\left.2 \mathrm{H}, 3^{\prime}-\mathrm{NH}_{2}, \mathrm{~d} 1\right), 6.32-6.36\left(\mathrm{~m}, 6 \mathrm{H}, \mathrm{H}-2^{\prime \prime}, 4^{\prime \prime}, 6^{\prime \prime}, \mathrm{d} 1+\mathrm{d} 2\right) \mathrm{ppm} .{ }^{13} \mathrm{C}-\mathrm{NMR}\left(75 \mathrm{MHz}, \mathrm{CDCl}_{3}\right): \delta=11.7,11.92,11.95$, $11.97,18.6,21.1,21.3,22.5,22.8,23.80,23.84,24.2,28.0,28.2,30.46,30.52,31.45,31.49,35.0,35.32,35.36$, $35.40,35.7,35.8,36.1,39.5,39.8,39.9,41.5,42.0,42.2,42.4,43.6,53.2,53.5,55.31,55.34,56.19,56.24$, 60.9, 61.0, 98.6 (C-4"), d1), 99.1 (C-4", d2), 106.05 (C-2", 6", d2), 106.08 (C-2"',6", d1), 108.0 (C-2, d1), 108.3 (C2, d2), 120.0 (CN, d1), 120.2 (CN, d2), 123.9 (C-1" , d1), 124.0 (C-1"', d2), 141.6 (C-3, d1), 141.8 (C-3, d2), 145.7, 145.8, 158.8 (C-3', d2), 158.9 (C-3', d1), 160.87 (C-3"',5", d2), 160.91 (C-3", 5', d1) ppm. HRMS (ESI ): m/z $[\mathrm{M}+\mathrm{H}]^{+}$calcd for $\mathrm{C}_{39} \mathrm{H}_{57} \mathrm{~N}_{2} \mathrm{O}_{3}: 601.4364$; found 601.4308 .

General procedure for the synthesis of compounds $(\mathbf{5 b}, \mathbf{c})$. Using the same standard experimental procedure described for compounds $\mathbf{4 b}$ and $\mathbf{4 c}$, employing 10 or 20 equiv of malononitrile.

(1R,3aS,3bR,5aS,11aS,11bS,13aR)-8-Amino-11a,13a-dimethyl-1-[(R)-6-methylheptan-2-yl]-10-(4methylphenyl)-2,3,3a,3b,4,5,5a,6,11,11a,11b,12,13,13a-tetradecahydro-1H-cyclopenta[c]tetraphene-7,9dicarbonitrile (5b) (19 mg, 33\%), mp 197-199 ${ }^{\circ} \mathrm{C} .{ }^{1} \mathrm{H}-\mathrm{NMR}\left(300 \mathrm{MHz}, \mathrm{CDCl}_{3}\right): \delta=0.61\left(\mathrm{~s}, 3 \mathrm{H}, 18-\right.$ or $\left.19-\mathrm{CH}_{3}\right)$, $0.62\left(\mathrm{~s}, 3 \mathrm{H}, 18\right.$ - or 19- $\left.\mathrm{CH}_{3}\right), 0.84-1.94(\mathrm{~m}, 34 \mathrm{H}), 1.91(\mathrm{~d}, 1 \mathrm{H}, \mathrm{H}-1, J 16.5 \mathrm{~Hz}), 2.34(\mathrm{~d}, 1 \mathrm{H}, \mathrm{H}-1, J 16.5 \mathrm{~Hz}), 2.41$ (s, $\left.3 \mathrm{H}, 4^{\prime \prime}-\mathrm{CH}_{3}\right), 2.54$ (dd, $1 \mathrm{H}, \mathrm{H}-4, J 18.8,12.0 \mathrm{~Hz}$ ), 2.91 (dd, $1 \mathrm{H}, \mathrm{H}-4, J$ 18.8, $5.4 \mathrm{~Hz}$ ), 4.98 (s, 2H, NH2), 7.04-7.11 (m, 2H, H-2" $\left.6^{\prime \prime}\right), 7.23-7.30$ (m, 2H, H-3", $\left.5^{\prime \prime}\right)$ ppm. ${ }^{13} \mathrm{C}-\mathrm{NMR}\left(75 \mathrm{MHz}, \mathrm{CDCl}_{3}\right): \delta=11.5,11.9,18.6,20.9,21.4$, 22.6, 22.8, 23.8, 24.2, 28.0, 28.2, 28.3, 31.4, 33.9, 34.9, 35.4, 35.8, 36.1, 39.5, 39.8, 40.8, 41.3, 42.3, 53.4, 56.3, 95.8 (C-4'), 96.9 (C-2'), 115.5 (CN), 115.9 (CN), 125.7 (C-2), 128.0 and 128.2 (C-2" and C-6"'), 129.3 and 129.6 (C-3" and C-5"), 134.1 (C-1"), 138.6 (C-4"), 146.0 (C-3), 149.5 (C-3'), 150.9 (C-1') ppm. HRMS (ESI ): m/z $[\mathrm{M}+\mathrm{Na}]^{+}$calcd for $\mathrm{C}_{40} \mathrm{H}_{53} \mathrm{~N}_{3} \mathrm{Na}$ : 598.4132; found 598.4149.

$(1 R, 3 a S, 3 b R, 5 a S, 11 a S, 11 b S, 13 a R)-8-A m i n o-10-(4-m e t h o x y p h e n y l)-11 a, 13 a-d i m e t h y l-1-[(R)-6-m e t h y l h e p t a n-$ 2-yl]-2,3,3a,3b,4,5,5a,6,11,11a,11b,12,13,13a-tetradecahydro-1H-cyclopenta[c]tetraphene-7,9-dicarbonitrile (5c) $(22 \mathrm{mg}, 37 \%), \mathrm{mp} 236-239{ }^{\circ} \mathrm{C} .{ }^{1} \mathrm{H}-\mathrm{NMR}\left(300 \mathrm{MHz}, \mathrm{CDCl}_{3}\right): \delta=0.61\left(\mathrm{~s}, 3 \mathrm{H}, 18\right.$ - or $\left.19-\mathrm{CH}_{3}\right), 0.62(\mathrm{~s}, 3 \mathrm{H}, 18-$ or 19- $\left.\mathrm{CH}_{3}\right), 0.68-1.95(\mathrm{~m}, 34 \mathrm{H}), 1.92(\mathrm{~d}, 1 \mathrm{H}, \mathrm{H}-1, \mathrm{~J} 16.5 \mathrm{~Hz}), 2.34(\mathrm{~d}, 1 \mathrm{H}, \mathrm{H}-1, J 16.5 \mathrm{~Hz}), 2.54(\mathrm{dd}, 1 \mathrm{H}, \mathrm{H}-4, J 18.8$, $12.0 \mathrm{~Hz}$ ), 2.91 (dd, $1 \mathrm{H}, \mathrm{H}-4, J$ 18.8, $5.4 \mathrm{~Hz}$ ), 3.87 (s, 3H, 4"'-OCH $\mathrm{H}_{3}, 4.98\left(\mathrm{~s}, 2 \mathrm{H}, \mathrm{NH}_{2}\right), 6.95-7.02\left(\mathrm{~m}, 2 \mathrm{H}, \mathrm{H}-3^{\prime \prime}, 5^{\prime \prime}\right)$, 7.08-7.16 (m, 2H, H-2" ,6") ppm. ${ }^{13} \mathrm{C}-\mathrm{NMR}\left(75 \mathrm{MHz}, \mathrm{CDCl}_{3}\right): \delta=11.5,11.9,18.6,20.9,22.6,22.8,23.8,24.2$, 28.0, 28.2, 28.3, 31.4, 33.9, 34.9, 35.4, 35.7, 36.1, 39.5, 39.8, 40.8, 41.4, 42.3, 53.4, 55.3, 56.27, 56.29 , 95.8 (C4'), 97.1 (C-2'), 114.1 and 114.2 (C-2" and C-6"), $115.5(\mathrm{CN}), 116.0(\mathrm{CN}), 125.9$ (C-2), 129.2 (C-1"'), 129.6 and

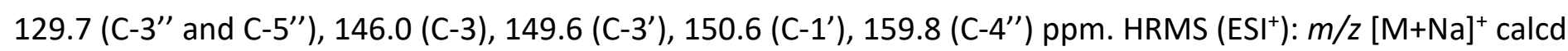
for $\mathrm{C}_{40} \mathrm{H}_{53} \mathrm{~N}_{3} \mathrm{ONa}$ : 614.4081; found 614.4096.

\section{Acknowledgements}

This work was supported by the University of Aveiro, Fundação para a Ciência e Tecnologia/Ministério da Ciência, Tecnologia e Ensino Superior, Centro2020 and Portugal2020, the COMPETE program, and the 
European Union (FEDER program) via the financial support to the LAQV-REQUIMTE (UIDB/50006/2020) research projects. PAGE project "Protein aggregation across the lifespan" (CENTRO-01-0145-FEDER-000003) supports Hélio M. T. Albuquerque Post-Doctoral grant (BPD/UI98/4861/2017).

\section{Supplementary Material}

${ }^{1} \mathrm{H}-,{ }^{13} \mathrm{C}-\mathrm{NMR}$ and $\mathrm{HRMS}$ spectra for all new compounds are available in the supplementary file accompanying this paper.

\section{References}

1. Cerqueira, N. M. F. S. A.; Oliveira, E. F.; Gesto, D. S.; Santos-Martins, D.; Moreira, C.; Moorthy, H. N.; Ramos, M. J.; Fernandes, P. A. Biochemistry 2016, 55, 5483.

https://doi.org/10.1021/acs.biochem.6b00342

2. Nes, W. D. Chem. Rev. 2011, 111, 6423.

https://doi.org/10.1021/cr200021m

3. Albuquerque, H. M. T.; Santos, C. M. M.; Silva, A. M. S. Molecules 2019, 24, 116.

https://doi.org/10.3390/molecules24010116

4. Zhao, L.; Chen, X.-J.; Zhu, J.; Xi, Y.-B.; Yang, X.; Hu, L.-D.; Ouyang, H.; Patel, S. H.; Jin, X.; Lin, D.; Wu, F.; Flagg, K.; Cai, H.; Li, G.; Cao, G.; Lin, Y.; Chen, D.; Wen, C.; Chung, C.; Wang, Y.; Qiu, A.; Yeh, E.; Wang, W.; Hu, X.; Grob, S.; Abagyan, R.; Su, Z.; Tjondro, H. C.; Zhao, X.-J.; Luo, H.; Hou, R.; Jefferson, J.; Perry, P.; Gao, W.; Kozak, I.; Granet, D.; Li, Y.; Sun, X.; Wang, J.; Zhang, L.; Liu, Y.; Yan, Y.-B.; Zhang, K. Nature 2015, 523, 607.

https://doi.org/10.1038/nature14650

5. Makley, L. N.; McMenimen, K. A.; DeVree, B. T.; Goldman, J. W.; McGlasson, B. N.; Rajagopal, P.; Dunyak, B. M.; McQuade, T. J.; Thompson, A. D.; Sunahara, R.; Klevit, R. E.; Andley, U. P.; Gestwicki, J. E. Science 2015, 350,674 .

https://doi.org/10.1126/science.aac9145

6. Yang, X.; Chen, X.-J.; Yang, Z.; Xi, Y.-B.; Wang, L.; Wu, Y.; Yan, Y.-B.; Rao, Y. J. Med. Chem. 2018, $61,8693$. https://doi.org/10.1021/acs.jmedchem.8b00705

7. Zhou, H.; Yang, Z.; Tian, X.; Chen, L.; Lee, S.; Huynh, T.; Ge, C.; Zhou, R. ACS Chem. Neurosci. $2019,10,4051$. https://doi.org/10.1021/acschemneuro.9b00285

8. Morzycki, J. W. Steroids 2014, 83, 62.

https://doi.org/10.1016/j.steroids.2014.02.001

9. Kemnitzer, W.; Drewe, J.; Jiang, S.; Zhang, H.; Crogan-Grundy, C.; Labreque, D.; Bubenick, M.; Attardo, G.; Denis, R.; Lamothe, S.; Gourdeau, H.; Tseng, B.; Kasibhatla, S.; Cai, S. X. J. Med. Chem. 2008, 51, 417. https://doi.org/10.1021/jm7010657

10. El-Far, M.; Elmegeed, G. A.; Eskander, E. F.; Rady, H. M.; Tantawy, M. A. Eur. J. Med. Chem. 2009, $44,3936$. https://doi.org/10.1016/i.ejmech.2009.04.020

11. Raghuvanshi, D. S.; Verma, N.; Singh, S.; Luqman, S.; Chand Gupta, A.; Bawankule, D. U.; Tandon, S.; Nagar, A.; Kumar, Y.; Khan, F. New J. Chem. 2018, 42, 16782. 
12. Zheng, J.; He, M.; Xie, B.; Yang, L.; Hu, Z.; Zhou, H.-B.; Dong, C. Org. Biomol. Chem. 2018, 16, 472. https://doi.org/10.1039/C7OB02794J

13. Auria-Luna, F.; Fernández-Moreira, V.; Marqués-López, E.; Gimeno, M. C.; Herrera, R. P. Sci. Rep. 2020, 10, 11594.

https://doi.org/10.1038/s41598-020-68076-1

14. Kumar, D.; Reddy, V. B.; Sharad, S.; Dube, U.; Kapur, S. Eur. J. Med. Chem. 2009, 44, 3805.

This paper is an open access article distributed under the terms of the Creative Commons Attribution (CC BY) license (http://creativecommons.org/licenses/by/4.0/) 